\title{
EchoGéo
}

$57 \mid 2021$

Les enjeux de l'alimentation en eau potable des villes

\section{Donner à voir l'empreinte territoriale d'un itinéraire culturel et de ses circulations par l'image géographique associée au discours}

David Lagarde, Patricia Panegos et Sébastien Rayssac

\section{(2) OpenEdition}

Journals

Édition électronique

URL : https://journals.openedition.org/echogeo/22329

DOI : $10.4000 /$ echogeo.22329

ISSN : 1963-1197

Éditeur

Pôle de recherche pour l'organisation et la diffusion de l'information géographique (CNRS UMR 8586)

Référence électronique

David Lagarde, Patricia Panegos et Sébastien Rayssac, « Donner à voir l'empreinte territoriale d'un itinéraire culturel et de ses circulations par l'image géographique associée au discours », EchoGéo [En ligne], 57 | 2021, mis en ligne le 30 septembre 2021, consulté le 10 novembre 2021. URL : http:// journals.openedition.org/echogeo/22329; DOI : https://doi.org/10.4000/echogeo.22329

Ce document a été généré automatiquement le 10 novembre 2021.

EchoGéo est mis à disposition selon les termes de la licence Creative Commons Attribution - Pas d'Utilisation Commerciale - Pas de Modification 4.0 International (CC BY-NC-ND) 


\title{
Donner à voir l'empreinte territoriale d'un itinéraire culturel et de ses circulations par l'image géographique associée au discours
}

\author{
David Lagarde, Patricia Panegos et Sébastien Rayssac
}

\section{Introduction}

1 Les sentiers labellisés Chemins de Saint-Jacques-de-Compostelle n'ont cessé de se multiplier à travers l'Europe au cours des trente dernières années (Santos, 2017 ; Craviotto, 2018). Cette augmentation du nombre de chemins ruraux menant vers Saint-Jacques-deCompostelle reflète la revitalisation contemporaine de cet itinéraire de pèlerinage, puisant ses racines à l'époque médiévale.

2 Au cours de l'année 2020, une exposition itinérante (illustration 1) à caractère scientifique a été conçue par des chercheurs géographes mobilisés dans le programme GeoCompostelle $^{1}$ (Appel à Projets Readynov, Région Occitanie). Ce projet de recherche vise à améliorer les connaissances sur les mobilités vers Compostelle et l'impact de ces circulations sur les territoires traversés. En privilégiant la collecte de discours et l'observation in situ, le collectif a constitué un matériau permettant de mieux saisir les réalités contemporaines du phénomène jacquaire, de mettre en avant la diversité des profils des marcheurs et de leurs pratiques touristiques, tout en se recentrant sur les lieux autour desquels s'organisent ces circulations et qui permettent de saisir certaines dynamiques territoriales. 
Illustration 1 - Lancement de l'exposition à l'atrium de l'Université Toulouse - Jean Jaurès

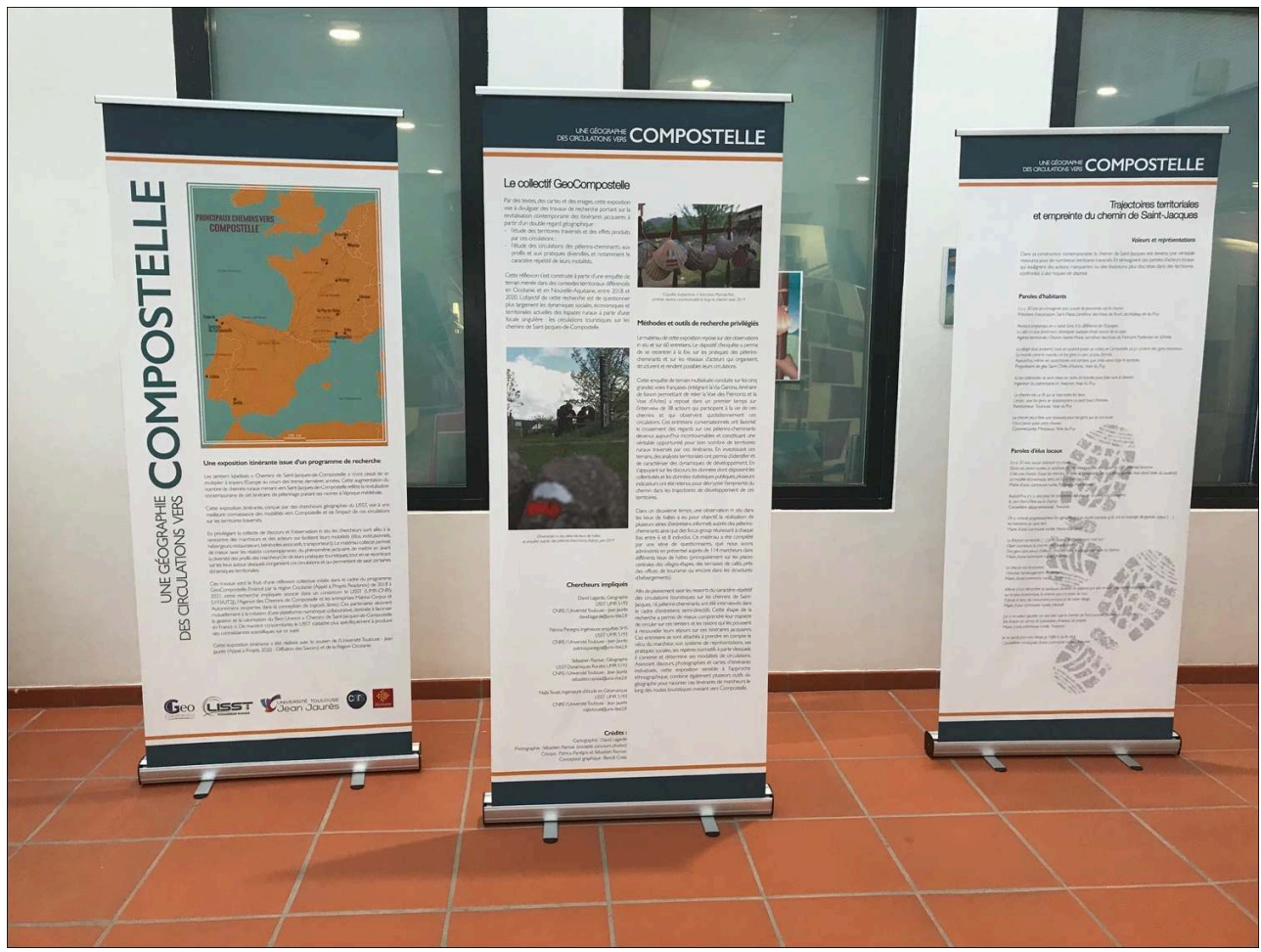

Source : LISST, S. Rayssac, novembre 2020

3 Cet article vise à questionner le processus de construction de l'exposition Une géographie des circulations vers Compostelle, basée sur la production d'une iconographie associée aux discours. À partir des propos collectés auprès de marcheurs et d'acteurs qui facilitent leurs mobilités, comment les images - ici géographiques - sont-elles élaborées et comment favorisent-elles la production d'un savoir scientifique et sa vulgarisation? En interrogeant le processus de construction de cette exposition, cet article :

- vise à rendre compte de la méthodologie de traduction de l'analyse de discours en images géographiques ${ }^{2}$;

- tout en expliquant comment l'image géographique peut également permettre à travers une description narrative, le passage d'un discours de sens commun à un discours scientifique.

4 En ayant en partage l'approche qualitative et la production de discours qui constitue le fil conducteur de cette exposition, notre collectif souhaite discuter de ce recours à l'image en privilégiant trois modalités d'association du discours à l'image :

- Dans sa construction contemporaine, le chemin de Saint-Jacques est devenu une véritable ressource pour de nombreux territoires traversés. Pour illustrer ce thème, la première partie place au cœur de l'interprétation la description narrative d'un élu local à partir d'un plan cadastral.

- L'itinérance jacquaire fait l'objet d'un engouement croissant. Avec l'augmentation de la fréquentation des itinéraires compostellans, on assiste à une diversification des profils de marcheurs, de leurs motivations, mais aussi de leurs pratiques. Pour nourrir ce thème, la deuxième partie place au cœur de la discussion la cartographie narrative pour témoigner du caractère répétitif des circulations vers Compostelle. 
- Le dernier volet de cette exposition donne la parole aux marcheurs et aux acteurs associatifs de ces chemins. Suite à l'organisation d'un concours photos, 27 photographies associées à des textes de témoignages ont été retenues. La troisième partie de cet article vise à questionner le processus de sélection opéré par les chercheurs ainsi que cette démarche qualifiée de "participative", tout en questionnant l'apport de ces images géographiques dans le corpus utilisé par le géographe pour alimenter et faire évoluer sa recherche.

\section{Décrypter la construction d'un itinéraire patrimonial et son empreinte territoriale}

5 Appuyé sur les discours, les données des collectivités territoriales et les données statistiques publiques, le premier volet de cette exposition aborde la construction de cet objet patrimonial - un chemin devenu ressource - et s'attache à décrypter son empreinte territoriale ${ }^{3}$. Plus qu'un simple itinéraire de randonnée, le chemin de SaintJacques-de-Compostelle peut constituer un levier pour le développement rural.

\section{Cerner les dynamiques de développement territorial à l'échelle d'une commune rurale par l'approche qualitative}

6 À l'origine de cette réflexion, différents modes de construction du matériau de recherche ont été sollicités: observation in situ, prises de notes descriptives, photographies des lieux, focus-group, questionnaires et enquêtes par entretiens. Ainsi le dispositif d'enquête par l'interview de 60 personnes a permis de se recentrer à la fois sur les pratiques des pèlerins-cheminants et sur les réseaux d'acteurs qui organisent, structurent et rendent possibles leurs circulations. Parmi eux, les entretiens semidirectifs de 38 acteurs $^{4}$ qui participent à la construction et à la vie de ces chemins ont constitué la base des analyses territoriales ayant pour objet l'identification et la caractérisation des dynamiques de développement à l'échelle des territoires ruraux.

7 La définition des territoires d'enquête et le recensement des acteurs à interviewer ont été des étapes décisives avant d'investir le terrain, de collecter les données et de proposer une grille d'analyse commune à l'ensemble des territoires enquêtés. Après l'interprétation des données, notre démarche a consisté dans cette exposition à privilégier l'exemple d'un territoire précis pour donner à voir au travers d'une sélection de verbatim, l'importance que revêt ce chemin pour certains territoires ruraux.

8 Pour comprendre comment cet itinéraire s'est construit, à partir de quels lieux patrimoniaux, de quelles actions et autour de quels acteurs, un cadre méthodologique a été défini pour la mise en œuvre et l'analyse des entretiens effectués. De précédents travaux nous avaient permis de mettre en lumière un système d'acteurs complexe lié aux chemins de Saint-Jacques (Rayssac et al., 2017) sur lesquels nous nous sommes appuyés pour la définition d'une typologie des acteurs à interviewer. L'énoncé d'un questionnement différencié par catégories d'acteurs (Gumuchian et al., 2003), a précédé l'élaboration de guides d'entretien qui portaient sur la trajectoire de l'acteur, les perceptions du territoire vécu (Di Méo, 1996), les liens et les représentations de l'acteur avec le phénomène jacquaire. 
9 Nous avons décidé de conduire cette enquête de terrain, située sur la région Occitanie, et dans une moindre mesure sur un département de la Nouvelle Aquitaine, sur différents sites des cinq grandes voies jacquaires françaises ${ }^{5}$, afin d'identifier les problématiques communes à ces itinéraires compostellans qui ont cependant des niveaux d'impacts territoriaux forts différents. Nous avons concentré notre travail sur la voie du Puy, qui de par l'ancienneté de sa structuration par les acteurs territoriaux et ses niveaux élevés de fréquentation permet d'opérer des analyses approfondies fondées sur le recul nécessaire pour appréhender l'empreinte du chemin dans les trajectoires de développement des territoires traversés.

Situé sur la voie du Puy, dans le département de l'Aveyron qui est particulièrement investi autour de ces chemins, dans une portion de sentier constitutive du bien culturel inscrit sur la Liste du patrimoine mondial, Saint-Côme-d'olt ${ }^{6}$ a été retenue comme commune d'intérêt pour l'exposition. Par sa configuration, ses attributs et ses actions, ce territoire nous a semblé représentatif des dynamiques territoriales à l'œuvre. À travers l'observation de cette commune rurale, le recours à l'analyse multiscalaire fut utile pour fonder notre analyse territoriale et prendre en compte les projets de territoires aux échelles intercommunales, départementales et régionales.

\section{De la collecte du discours à l'utilisation de l'image}

11 Parmi la série d'entretiens conversationnels effectuée dans cette recherche, les échanges avec le maire de Saint-Côme-d'olt ont constitué pour nous un temps fort de l'enquête. D'une part, le caractère illustratif de la description narrative du maire conforta notre démonstration. D'autre part, le recours inattendu à un plan cadastral (illustration 2) lors de l'élaboration par l'élu local de son discours démonstratif, interroge le chercheur sur l'utilisation d'une image géographique pour construire un discours de sens commun (Mucchielli, 2006; Bardin, 2007) et donner à voir l'information géographique. 
Illustration 2 - Description narrative du maire et outil d'interprétation à partir du plan cadastral du centre-bourg, Saint-Côme-d'Olt

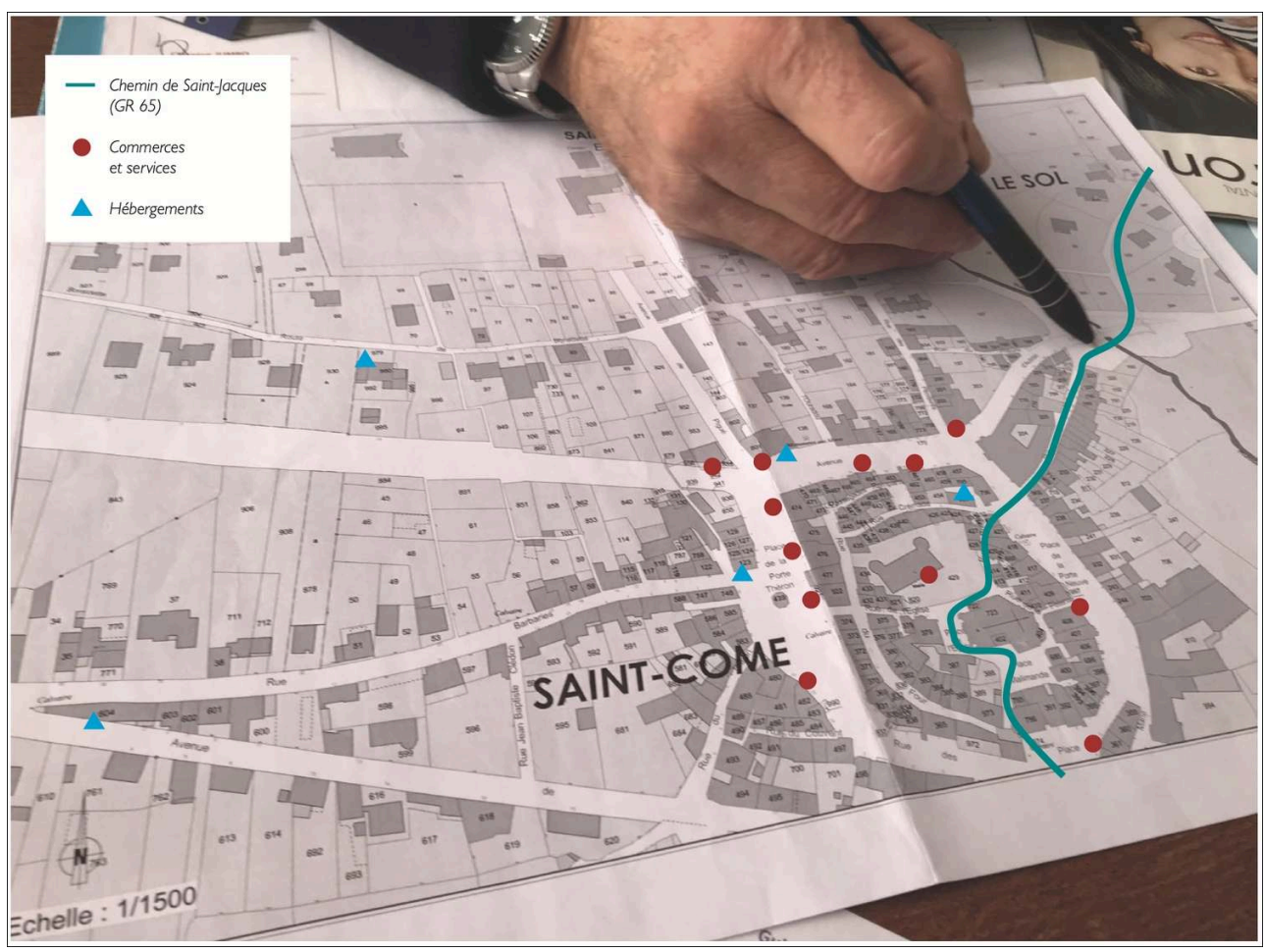

Source : LISST, P. Panegos et S. Rayssac, octobre 2020.

Dans le cadre de cet entretien effectué à deux chercheurs (avec enregistrement et prises de notes) dans le bureau du maire, l'image - ici le plan cadastral du centrebourg - a été mobilisée spontanément par l'élu rural pour appuyer son discours sur les impacts des chemins de Saint-Jacques pour sa commune et les territoires de projets évoqués. Le recours à l'image fut pour le maire un moyen d'aider à formuler, structurer et illustrer son argumentaire. Au cours de l'entretien, l'ensemble des thèmes prédéfinis avant la collecte de données ont été nourris et illustrés par des exemples concrets, relatés par l'élu et identifiés par autant de tracés et de points - ici éphémères car non écrits - sur le plan cadastral. Au-delà des données informatives collectées, c'est bien un examen de cette figure visuelle (Lussault, 2013) se dessinant sous nos yeux, de cette vision subjective, qu'il a fallu ensuite engager. Sans modifier le déroulement de cet entretien (Blanchet et Gotman, 1992), le son associé à une captation vidéo aurait certainement permis de faciliter la reconstitution de cette information plurielle et l'analyse de contenu. Cette dernière a d'ailleurs consisté au travers de la grille que nous avions définie à faire ressortir les différents thèmes identifiés, pour les associer, les hiérarchiser et finalement produire un cadre d'observation des impacts sur le territoire. Ce cadre a été complété par une recherche sur les données du territoire.

\section{Le plan cadastral comme élément d'interprétation et de visualisation des données de l'analyse scientifique}

Après la phase d'analyse et d'interprétation des données, nous avons retenu dans cette recherche sept thématiques permettant de qualifier l'impact des chemins sur les territoires, à savoir: réaménagement de l'espace public, stabilité démographique, 
immobilier actif, maintien de l'économie, consolidation des services, développement de projets de territoires, valeurs patrimoniale et intellectuelle. Dans le cadre de l'exposition, nous avons requis l'aide de l'image en sollicitant la carte de la commune pour illustrer ces éléments au grand public. Pour cela, nous avons utilisé la photographie du plan cadastral (mobilisé lors de l'entretien par le maire) comme « fond d'image " sur laquelle nous avons fait figurer des informations (commerces, services, gîtes, emprise du chemin, etc.) qui illustrent les propos du maire et les données complémentaires que nous avons apportées. Le cadastre est « l'une des grandes sources d'information du géographe» (Brunet, Ferras et Théry, 1992, p. 79). En évoquant ces parcelles de propriétés, numérotées, le maire a identifié avec précision le bâti rénové, l'espace public réhabilité, la création de certains commerces, la reprise d'activité avec le changement de propriétaire d'un édifice ou d'une entreprise, les lieux fréquentés, les services proposés aux marcheurs, en résumé toute une géographie où l'empreinte du chemin est clairement lisible et fait apparaitre l'importance de cet itinéraire patrimonial et touristique pour ces territoires ruraux. Ici le visuel que nous avons commenté et illustré de verbatim, vient donc offrir une lecture rapide du propos scientifique développé à partir d'un discours recueilli.

\section{Cartographier les circulations pédestres vers Compostelle}

14 Afin d'illustrer la diversité des circulations pédestres observées sur les chemins de Compostelle, le deuxième volet de cette exposition est composé de cartes d'itinéraires individuels (Besse, 2010), basé sur un matériau narratif collecté auprès de pèlerinscheminants.

\section{Une cartographie narrative élaborée sur la base de récits de voyage}

Les cartes d'itinéraires présentées dans l'exposition Une géographie des circulations vers Compostelle sont basées sur des récits de voyages de pèlerins-cheminants, obtenus dans le cadre d'entretien semi-directifs qui se sont déroulés en deux temps. La première phase de collecte a eu lieu sur les chemins de Saint-Jacques, au printemps 2019. En mars et avril 2020, nous avons ensuite recontacté les personnes enquêtées, en vue d'obtenir des informations plus précises et détaillées sur leurs voyages. Cette démarche cartographique se rapproche en cela du courant de la cartographie narrative (Caquard et Cartwright, 2014; Fournier, 2016). Elle se situe plus précisément dans le prolongement de travaux récents, ayant conduit à la mise en carte de récits portant sur des formes et des échelles de mobilités variées: trajectoires migratoires transnationales (Bacon, 2016; Mekdjian et al., 2014), espaces vécus du quotidien ((Olmedo, 2011), ou encore parcours urbains fictifs tirés de romans policiers (Troin, 2017). L'élaboration de cartes mettant en lumière des circulations touristiques sur les chemins de Compostelle s'inscrit aussi dans la continuité d'une réflexion portant sur la cartographie des mobilités individuelles, engagée dans le cadre de recherches précédentes, à partir du cas des parcours de réfugiés syriens vers la Jordanie (Lagarde, 2020).

16 Durant les entretiens, les questions adressées aux enquêtés visaient à leur faire générer des récits de voyage portant sur chacun des séjours qu'ils ont effectués sur les chemins 
de Compostelle. Au cours de ces échanges, nous apportions systématiquement une attention particulière à la spatialisation de ces expériences de mobilités : points de départ et d'arrivée, toponymes des étapes, dates des différents séjours, modes d'hébergement, etc. Au-delà des seules informations obtenues dans le cadre de ces entretiens, nous nous sommes également appuyés sur les carnets de bord que certains de nos interlocuteurs rédigeaient sur la route. Ce matériau s'est avéré particulièrement précieux afin d'obtenir des informations détaillées sur des voyages parfois longs de plusieurs milliers de kilomètres.

17 Une fois ce matériau narratif réuni, nous nous sommes appliqués à transformer ces récits de voyages en « bases de données cartographiables » (Caquard et Joliveau, 2016), en appliquant des figurés spécifiques aux différents moyens de locomotion empruntés, aux divers types d'hébergement choisis, ou encore au temps passé dans chacune des étapes du voyage. Étant soumis à des contraintes de temps liées aux délais de restitution du contenu final de l'exposition à remettre au graphiste et à l'imprimeur, nous n'avons pas été en mesure de traiter l'ensemble du matériau collecté de manière optimale. En effet, seul le récit de voyage de Céline a pu faire l'objet d'un traitement cartographique abouti. La suite de cette contribution se focalisera donc sur le parcours de cette enquêtée.

\section{Les pérégrinations de Céline}

Céline est âgée de 34 ans. En 2017, elle décide de prendre un congé sans solde pour voyager. En quête de spiritualité et d'aventure, elle commence par un séjour de deux semaines sur les chemins de Compostelle, au départ de St-Jean-Pied-de-Port. Là, elle fait la connaissance d'une femme qui lui parle d'ayurvéda et de yoga. Elle-même avait parcouru le chemin de Saint-Jacques avant de partir vivre quelques temps en Inde. Cette rencontre incite Céline à partir à son tour dans ce pays, pour se former aux techniques de massages ayurvédiques. Quelques mois après son retour, elle décide de revenir sur les chemins de Saint-Jacques, en partant cette fois-ci du Puy-en-Velay (illustration 3). En échange du gîte et du couvert, elle propose des massages thérapeutiques aux personnes rencontrées sur sa route. 
Illustration 3 - Les pérégrinations de Céline : Le Puy-Lexos (5 au 27 juin 2019)

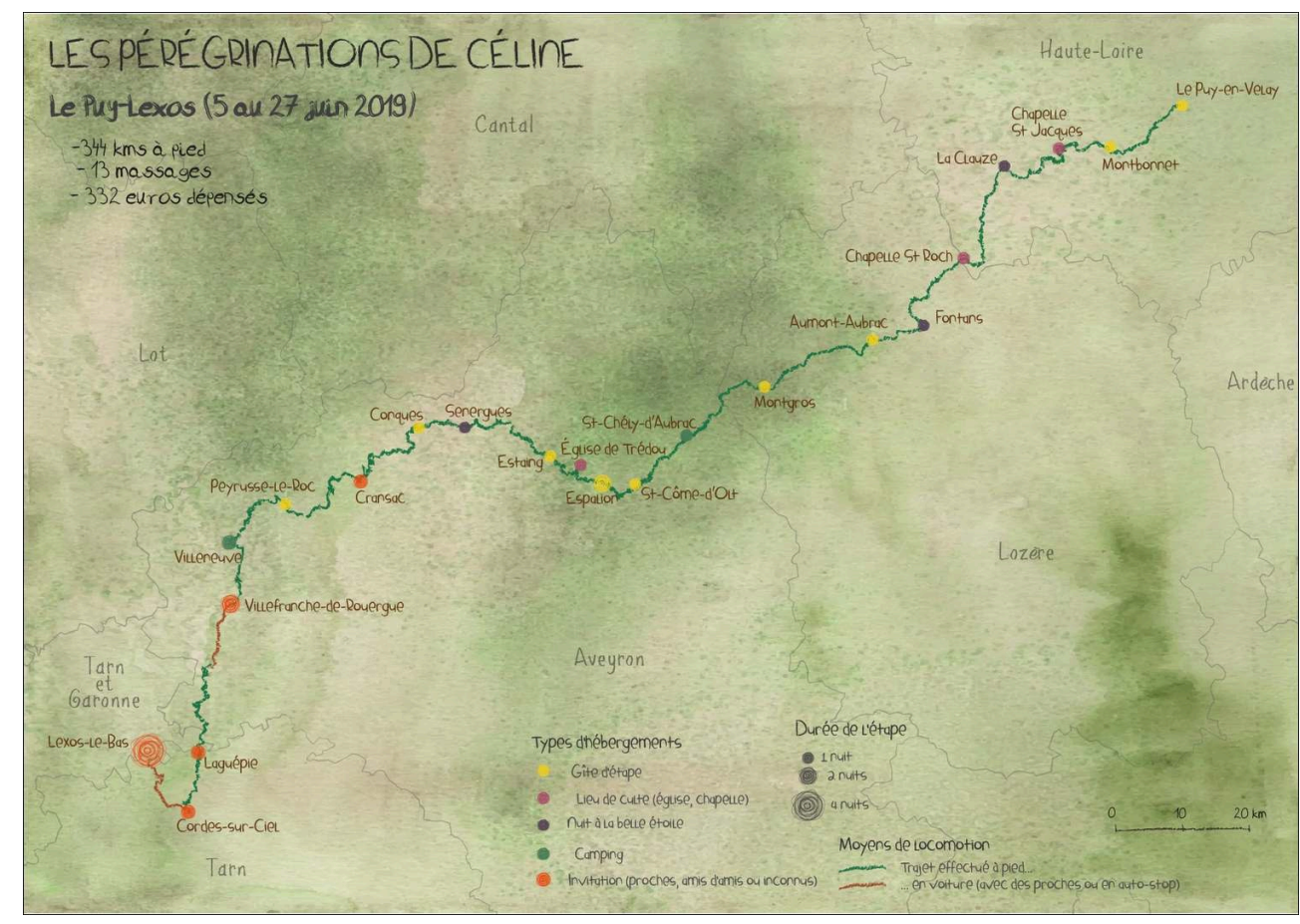

Source : LISST, D. Lagarde, octobre 2020

19 Céline alterne entre de longues journées de marche et plusieurs jours passés au même endroit. Sur les cartes de son parcours, cette dimension est représentée sous la forme de cercles proportionnels, plus ou moins grands en fonction de la durée passée dans chacune des étapes. Les couleurs de ces figurés ponctuels permettent pour leur part de distinguer les localités dans lesquelles Céline a dormi à la belle étoile, dans des gîtes, des chapelles ou encore chez des amis ou des inconnus rencontrés chemin faisant. Sur ce point, la lecture des cartes des pérégrinations de Céline permet de constater que les modes d'hébergement qu'elle privilégie évoluent au fur et à mesure de sa progression dans l'espace. En effet, alors qu'elle opte pour le bivouac comme alternative aux gîtes durant les premiers jours suivant son départ du Puy, plus le temps passe et plus elle semble à l'aise à l'idée de s'ouvrir aux autres, d'être accueillies chez des inconnus (illustration 4). Pour Céline, emprunter les chemins de Compostelle répond en effet à une envie d'échanger avec les personnes rencontrées sur la route, au hasard du chemin. 
Illustration 4 - Les pérégrinations de Céline : Lexos - Lourdes (28 juin au 18 juillet 2019)

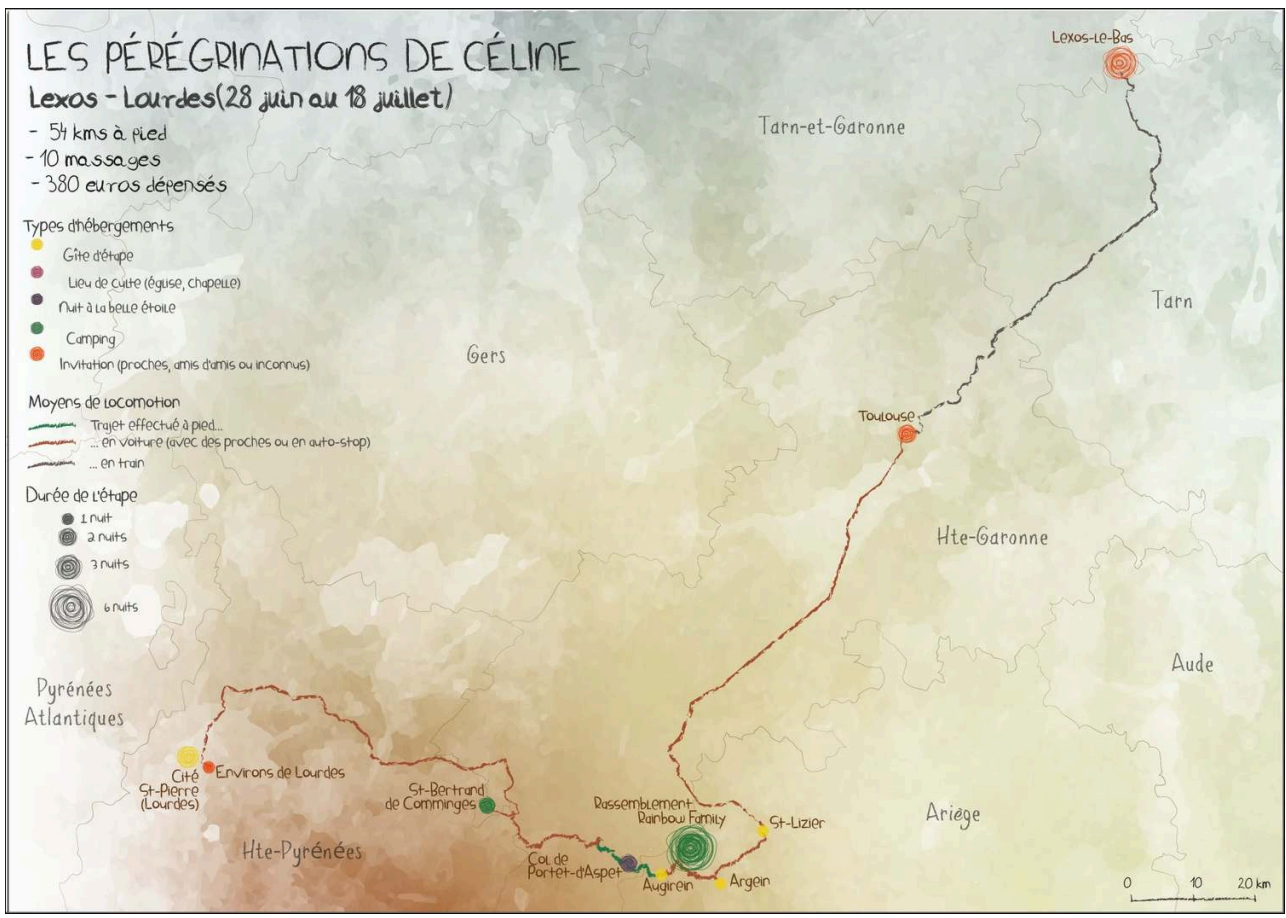

Source : LISST, D. Lagarde, octobre 2020.

Sur le plan géographique, elle estime que cet itinéraire concentre "une énergie particulière, parce qu'il passe par plein d'églises, de points spécifiques, religieux et spirituels (...) qui le rendent différent des autres chemins de randonnée au niveau spirituel ». Ces 69 jours passés sur la route à pied, en train, en voiture et en bus différents moyens de transports représentés par des tracés de couleurs différentes les lieux qu'elle a pratiqués et les rencontres qu'elle y a effectuées ont profondément nourri ses envies de reconversion professionnelle. Aujourd'hui, Céline aspire à vivre en pleine nature "à plusieurs, en cultivant une autonomie alimentaire et énergétique ». Comme elle le dit elle-même, elle souhaite à présent « diffuser les valeurs du yoga et de la spiritualité, éveiller les gens et créer [son] petit paradis ». 


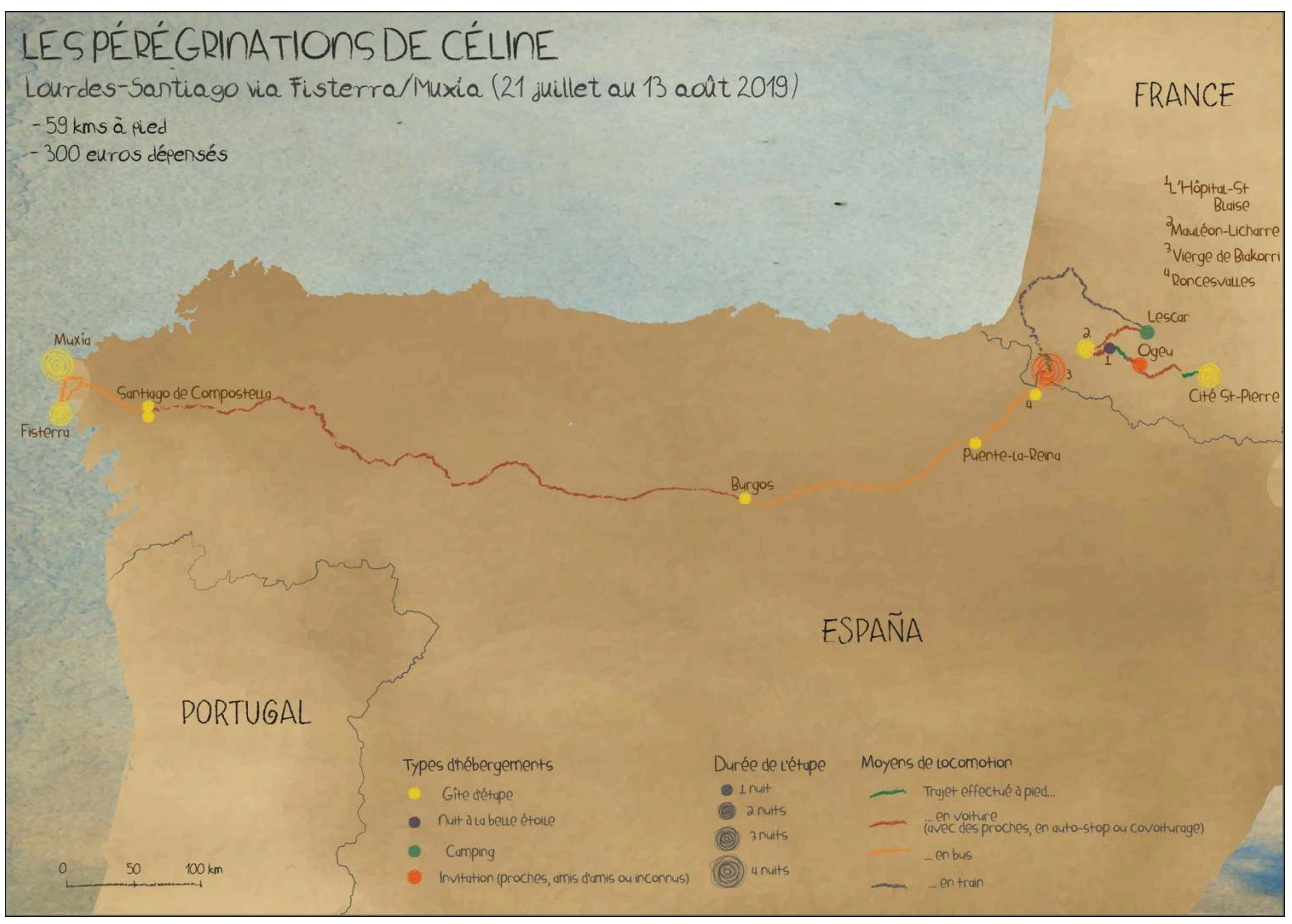

Source : LISST, D. Lagarde, octobre 2020

L'enthousiasme de Céline à l'évocation de ses pérégrinations sur les chemins de Compostelle était perceptible tout au long de notre entretien. Afin de mieux retranscrire l'énergie et les émotions transmises durant nos échanges, nous avons choisi de dessiner des cartes aux couleurs vives et chaleureuses, qui rappellent la nature des paysages qu'elle a traversés jusqu'à St-Jacques : la verdure des plateaux de la Margeride et de l'Aubrac à la fin du printemps, qui contrastent avec l'aridité du hautplateau castillan de la Meseta (illustration 5), «écrasé » par le soleil du mois d'août. En outre, pour évoquer l'esprit du carnet de bord de Céline et des croquis griffonnés par certains voyageurs sur le bord des chemins, nous avons choisi d'opter pour des codes graphiques aux contours approximatifs, tout en utilisant des textures d'aquarelles. En ayant recours à ce procédé, notre intention était de « réintroduire les gens de la carte " (Rekacewicz et Tratnjek, 2016).

\section{Une approche artistique participative}

Dans le $3^{\text {ème }}$ volet de l'exposition, nous souhaitions recueillir des témoignages motivés par la pratique des chemins de Saint-Jacques et portant sur des émotions personnelles suscitées par la confrontation à cette route. Nous avons donc choisi de lancer un appel à participation, en vue de collecter des images accompagnées de textes évoquant la relation qu'entretiennent les contributeurs avec cet itinéraire culturel, souvent qualifié de « route mythique » (Musset, 2018). 


\section{Collecte et processus de sélection des contributions}

Pour mener à bien cet aspect notre projet, nous nous sommes coordonnés avec notre partenaire institutionnel, l'Agence des Chemins de Compostelle (ACIR). Celui-ci a adressé un premier message d'appel à contributions par voie de presse, dans le quotidien régional La Dépêche du Midi, avant de lancer une communication plus ciblée, via ses réseaux sociaux. Notons par ailleurs que l'appel a été lancé durant le premier confinement lié à la crise sanitaire au printemps 2020. Nous considérions en effet qu'il s'agissait là d'une période relativement propice à la collecte d'un tel matériau, dans la mesure où une majorité de personnes disposait de plus de temps libre que d'habitude, cela leur permettant de se replonger dans leurs souvenirs de voyage et de se consacrer à l'écriture d'un texte.

$\mathrm{Au}$ total, 83 personnes ont répondu à notre appel, en renvoyant entre une et cinq photographies chacune. La centaine de textes et de photographies reçus reflète, d'une part, le caractère particulièrement hétéroclite du public des pèlerins-cheminants et, d'autre part, le rapport que ces personnes entretiennent avec les territoires traversés. Au regard du nombre particulièrement important de messages reçus en un mois, nous avons ensuite opéré une sélection afin de choisir les contributions à intégrer à l'exposition. Notre grille de sélection s'est basée sur plusieurs critères : l'originalité des éléments envoyés, leur caractère représentatif de la manière de circuler sur les chemins et les traces laissées par ces circulations sur les territoires traversés. Un collectif composé de chercheurs (LISST) et d'institutionnels du patrimoine (Agence des chemins de Compostelle) a ensuite opéré une pré-sélection d'une trentaine de photographies, répondant à ces critères. Ce jury s'est finalement réuni pour confronter ce matériau et sélectionner in fine 27 textes et photographies - envoyés par 22 participants - qui ont été intégrés à l'exposition (illustration 6). 
Illustration - 6 : Les quatre panneaux valorisant les témoignages des pèlerins-cheminants

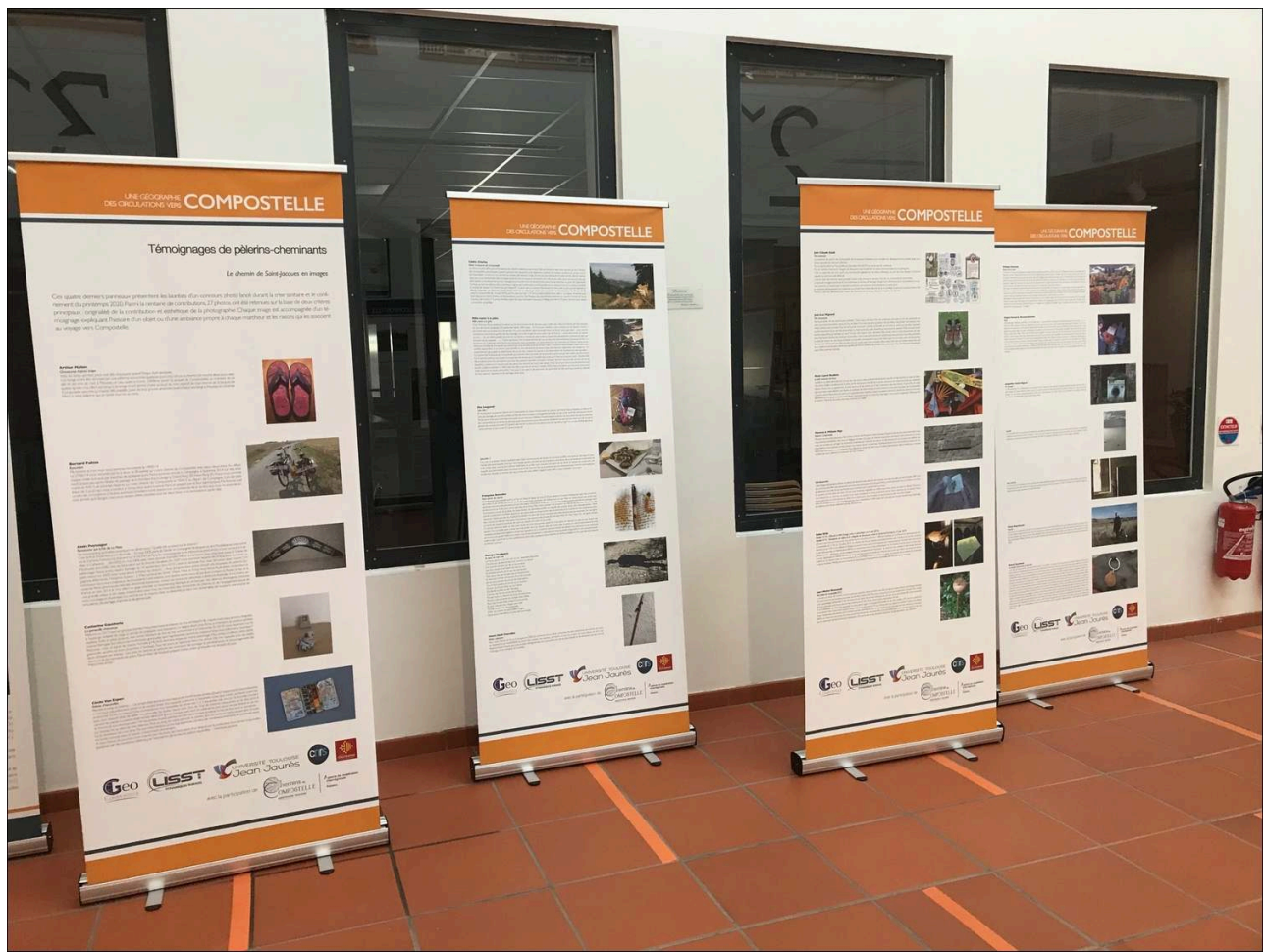

Source : LISST, S. Rayssac, novembre 2020

Nous avons choisi dans la suite du texte d'offrir un aperçu de cette troisième partie de l'exposition à travers deux exemples, qui nous semblent particulièrement évocateurs des dynamiques et des émotions générées par les chemins de Saint Jacques.

\section{Un regard sur l'action du tissu associatif local}

Si la grande majorité des retours de cet appel à exposition concerne des marcheurs, nous avons choisi, en guise d'ouverture, de présenter ici la contribution d'une association de bénévoles (illustration 7) qui pratiquent les chemins dans le cadre d'une activité de "loisirs " qui n'est pas la marche, et qui y trouvent pourtant un même potentiel de qualité relationnelle rencontré par les cheminants. Cet exemple montre également comment la prise de conscience et l'appropriation de la valeur patrimoniale des chemins peut transformer la population locale en véritable acteur territorial contribuant de manière active à faire vivre le chemin. 
Illustration 7 - Association Mille mains à la pâte

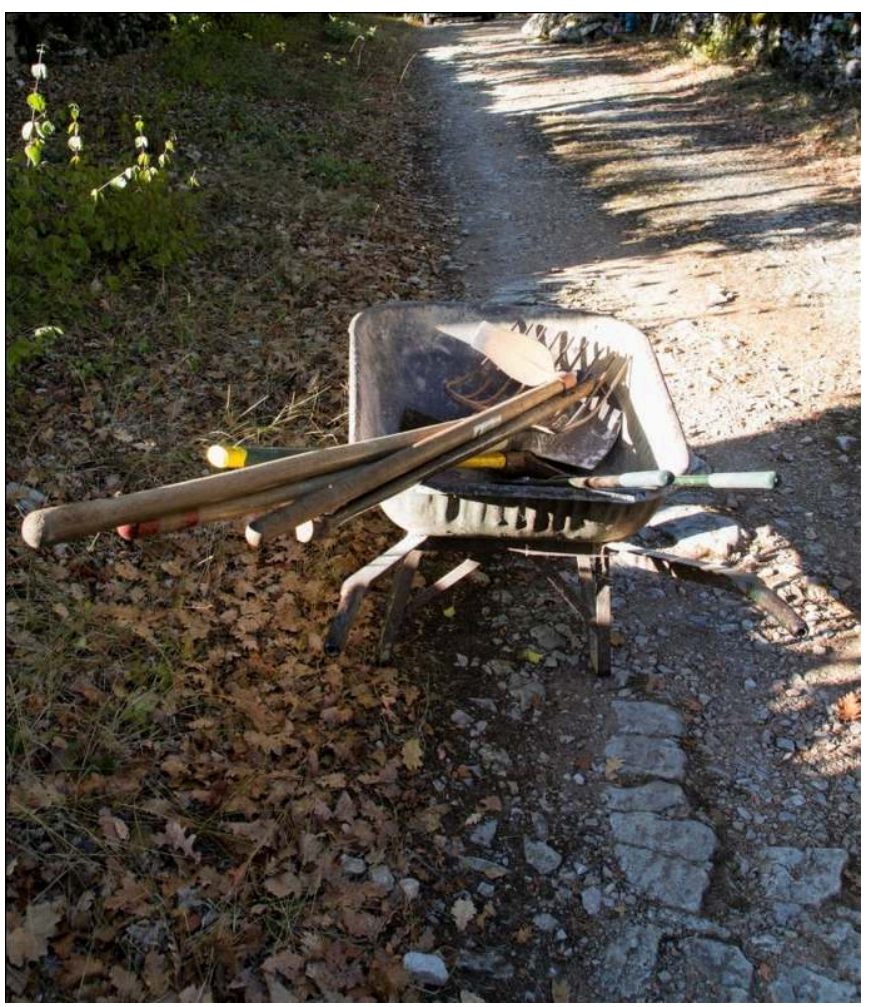

Source : Association Mille mains à la pâte, octobre 2020.

"C'est l'histoire d'une aventure humaine qui lie les hommes et les femmes pour mettre en valeur le chemin de Saint-Jacques sur leur territoire, quelques 500 bénévoles faisant 1000 mains... Ils n'ont pas marché au sens propre sur ce chemin, mais ils y ont passé beaucoup beaucoup de temps! Ils y ont sué, peiné, rigolé, échangé munis de force outils que voici ... pour que les nombreux cheminants gardent de leur passage une belle image de notre petit coin de France ...voire aient envie d'y revenir un jour.... Ils ont débroussaillé, reconstruit ces fameux murets de pierre sèche, réparé des éboulements, rénové du petit patrimoine (puits, cabanes, etc.). Notre territoire, c'est le département du Lot qui est riche de nombreux atouts qui en font un territoire où il fait bon vivre. Parmi ces richesses, nous possédons un patrimoine hors du commun, un chemin classé au patrimoine mondial de l'humanité que beaucoup nous envient, et en particulier trois des sept tronçons remarquables. Cette distinction confère aujourd'hui à ce chemin une visibilité et une attractivité certaines, mais elle implique également des exigences: un cheminement de qualité, la préservation de ce qui fait «l'esprit du chemin », la conservation de l'authenticité des paysages... Ce chemin de Saint-Jacques de Compostelle qui traverse notre territoire est emprunté tous les ans par des milliers de cheminants. Au fil des rencontres, tous louent le travail des bénévoles qui lui rendent peu à peu, au fil des ans, tout son lustre. Quelle belle récompense pour les participants qui, tous les premiers samedis d'octobre, viennent donner une journée de leur temps et perpétrer patiemment l'œuvre que nos aïeux ont façonné au cours des siècles. C'est ce à quoi nous nous employons tous les ans. La $6{ }^{\text {ème }}$ opération « 1 000 mains à la pâte » aura lieu le samedi 3 octobre 2020. Nous comptons sur tous pour poursuivre cette aventure en toute convivialité. C'est aussi à ces valeurs de rencontre, de générosité, de lien que nous sommes attachés et nous serions heureux de les partager avec vous ». 


\section{Un objet ordinaire des circulations compostellanes}

Le Bourdon, comme objet symbolique, mais surtout fidèle compagnon de tous les marcheurs depuis l'époque moyenâgeuse, est ici retenu dans ce deuxième témoignage. Cette photographie (illustration 8) montrant l'ombre du marcheur, ainsi anonymisé, marque son caractère universel. Le choix du texte, qui en appelle à la poésie, caractérise l'espace de créativité dont peut bénéficier le cheminant sur le temps du chemin. Cette réflexion personnelle relate par l'expérience la pénibilité ainsi que la recherche spirituelle liées aux chemins dans différents lieux géographiques.

Illustration 8 - Au bout de mes bras

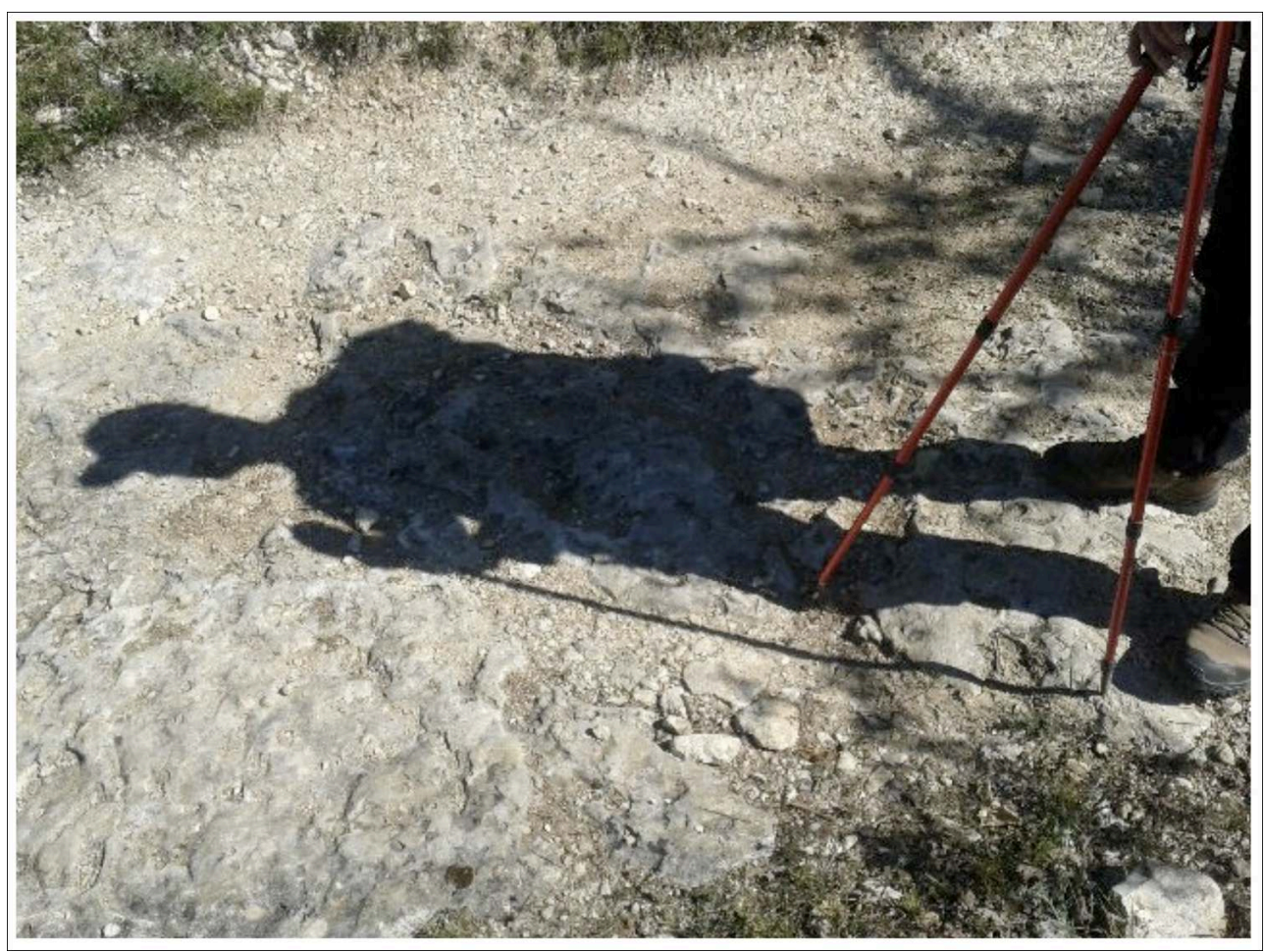

Source : Georges Esculpavit, octobre 2020

Certains, parce qu'ils n'en ont qu'un, l'appellent Bourdon. D'autres aux jambes fortes n'en auront nul besoin.

J'en connais certains qui tel un accordéon Les font sauter et virevolter de loin en loin. Et d'autres encore, sur le sac les porteront. Mais pour moi, qui ne suis pas un Amphitryon Et dont les mollets ont besoin d'un fort soutien Jamais ne pourrais me priver de mes bâtons Qui m'ont soutenu et porté sur le chemin. Partant du Puy en Velay ou de Fatima De Séville la belle ou de Hendaya Camino del Norte ou Via de la Plata, Portant la foi du chemin au bout de mes bras, Tordus, rayés, propres ou crottés mais fidèles Pour moi ils sont allés jusqu'à Compostelle, Et sans vraiment leur ériger une stèle Ni une cérémonie sacramentelle, 
Offrir du chemin, les plus belles images

Serait pour mes bâtons, le plus bel hommage. pour présenter deux exemples se montrait forcément subjective. Ces deux témoignages permettent par ailleurs d'illustrer la richesse que représente ce « corpus photograhique associé à un texte " que nous avons collecté, au départ dans une démarche sans intention scientifique, mais qui pourrait venir enrichir le matériau de recherche constitué initialement, pour prolonger notre analyse.

\section{Conclusion}

L'objectif initial de cette exposition itinérante vise à la diffusion d'une partie des résultats de notre recherche auprès de différents publics.

Dans ce projet, le recours à l'image nous a permis de créer un fil conducteur entre différentes approches géographiques qui, en les combinant, permettent de mieux restituer toute la richesse de ce sujet ainsi que la complexité des évolutions contemporaines du phénomène jacquaire et de leurs conséquences sur les territoires traversés. Ainsi, cette exposition a constitué le support de présentation de nos travaux à la communauté des chercheurs à l'occasion de plusieurs manifestations scientifiques. De la seule évocation jusqu'à la représentation, en passant par l'interprétation, le recours à l'image est plus que jamais mobilisé dans le vocabulaire géographique et, plus largement, dans l'ensemble des sciences humaines et sociales. Adossé au discours, son apport permet de rendre visibles des réalités sociales et territoriales. À travers cet article, nous souhaitions proposer une réflexion autour de la mobilisation de l'image dans le cadre d'une recherche géographique.

Illustration 9 - Présentation de l'exposition à la maison de l'Aubrac

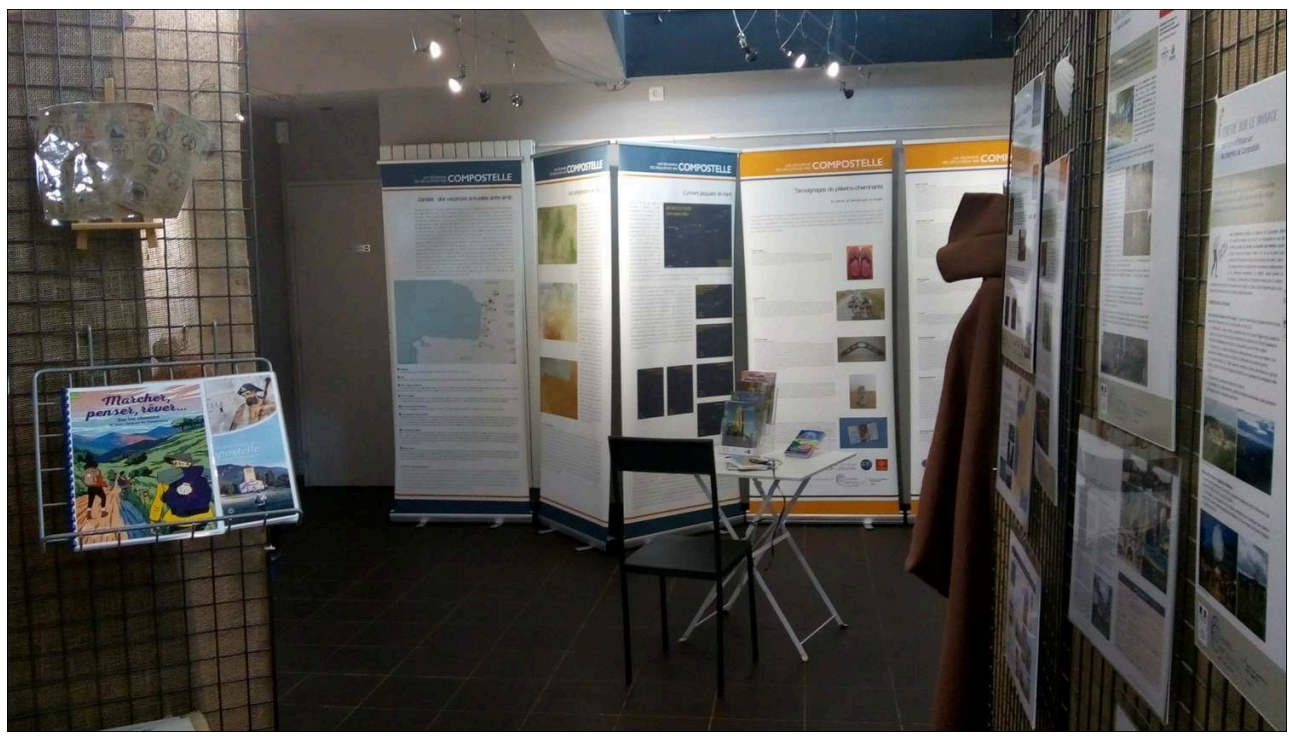

Source : Régine Pechberty, PNR de l'Aubrac, juin 2021.

En outre, ce projet permet aussi de faire un juste retour de nos résultats de recherche aux acteurs qui nous ont accordé du temps et de l'intérêt lors de nos enquêtes de terrain. Il s'agissait également de sensibiliser un public plus large aux réalités 
contemporaines du phénomène jacquaire, qui constitue une ressource mobilisable pour les territoires que nous avons observés. Dans cette perspective, cette exposition est d'ores-et-déjà accueillie sur de nombreux territoires traversés par les chemins de Compostelle (illustration 9). Cette diffusion des savoirs s'accompagne d'un travail de médiation scientifique que nous allons réaliser autour de cette exposition. Ces temps d'échanges favorisent ainsi le dialogue entre le chercheur et la société civile et permet de rendre les travaux de recherche plus accessibles, tout en confrontant une partie de nos résultats au-delà des seuls cercles universitaires. En privilégiant la dimension visuelle dans ce projet culturel, l'image géographique permet de " plonger » le public dans un univers et un espace-temps spécifiques qui deviennent plus tangibles voire plus ludiques que l'écrit, forme de restitution habituellement utilisée par la communauté scientifique.

\section{BIBLIOGRAPHIE}

Bacon L., 2016. La cartographie à l'épreuve de la complexité des parcours migratoires. Cartes et géomatique, $\mathrm{n}^{\circ} 229-230$, p. 147-166.

Bardin L., 2007. L'analyse de contenu. Paris, Presses Universitaires de France, 291 p.

Bertaux D., 1980. L'approche biographique : sa validité méthodologique, ses potentialités. Cahiers Internationaux de Sociologie, vol. 69, p. 197-225.

Besse J.-M., 2010. Cartographies. In Les carnets du paysage. Arles, Actes Sud, p. 5-9.

Blanchet A., Gotman A., 1992. L'enquête et ses méthodes : l'entretien. Paris, Nathan Université, 125 p.

Brunet R., Ferras R., Thery H., 1993. Les Mots de la géographie : Dictionnaire critique. La

Documentation française, Dynamiques des territoires, $518 \mathrm{p}$.

Caquard S., Cartwright W., 2014. Narrative Cartography: From Mapping Stories to the Narrative of Maps and Mapping. The Cartographic Journal, vol. 51, n 2, p. 101-106.

Caquard S., Joliveau T., 2016. Penser et activer les relations entre cartes et récits. Mappemonde, $\mathrm{n}^{\circ}$ 118, [En ligne]. URL: http://mappemonde.mgm.fr/118as1/

Craviotto L. P., 2018. Et l'Europe créa Compostelle. Paris, Le Monde diplomatique [En ligne]. URL: https://www.monde-diplomatique.fr/2018/08/CRAVIOTTO/58950

Di Méo G. (dir.), 1996. Territoire du quotidien. Paris - Montréal, L’Harmattan, 208 p.

Fournier M., 2016. Cartographier les récits. Clermont-Ferrand, Presses Universitaires Blaise Pascal, $242 \mathrm{p}$.

Gumuchian H., Grasset E., Lajarge R., Roux E., 2003. Les acteurs, ces oubliés du territoire. Paris, éd. Economica, Anthropos, $186 \mathrm{p}$.

Lagarde D., 2020. Construire une cartographie des expériences vécues. Récits migratoires et cartes d'itinéraires d'exilés syriens en Jordanie. Mappemonde [En ligne], n 129, URL: http:// journals.openedition.org/mappemonde/4568 
Lussault M., 2013. Image. In Levy J., Y.-M. (dir.), Dictionnaire de la géographie et de l'espace des sociétés. Paris, Belin, 1228 p.

Mekdjian S., Amilhat-Szary A.-L., Moreau M., Nasruddin G., Deme M., Houbey L., Guillemin C., 2014. Figurer les entre-deux migratoires. Pratiques cartographiques expérimentales entre chercheurs, artistes et voyageurs. Carnets de géographes [En ligne], $\mathrm{n}^{\circ}$ 7. URL: http:// journals.openedition.org/cdg/790

Mucchielli R., 2006. L'analyse de contenu des documents et des communications. Issy-les-Moulineaux, édition ESF, Série Développement personnel, $223 \mathrm{p}$.

Musset A., 2018. Routes mythiques. Quand l'histoire se mêle à la légende. Paris, Armand Colin, 159 p.

Olmedo E., 2011. Cartographie sensible, émotions et imaginaire. Visions carto, [En ligne]. URL: https://visionscarto.net/cartographie-sensible

Rayssac S. (dir.), 2017. Cheminements, tourisme et gouvernance territoriale. Sud-Ouest Européen [En ligne], $\mathrm{n}^{\circ}$ 43, 123 p. https://journals.openedition.org/soe/2494

Rekacewicz P., Tratnjek B., 2016. Cartographier les émotions. Carnets de géographes [En ligne], n 9. URL: http://journals.openedition.org/cdg/687

Santos X. M., 2017. La construction touristique des chemins de Saint-Jacques-de-Compostelle en Espagne : acteurs, regards et contradictions. Sud-Ouest Européen, n 43, p. 7-19.

Troin, F., 2017. Cartographie du Marseille d'un héros de roman policier (Total Khéops de J.-C. Izzo). Mappemonde [En ligne], $\mathrm{n}^{\circ}$ 121. URL: http://mappemonde.mgm.fr/121_as2/

\section{NOTES}

1. Cette exposition est le fruit des recherches qui ont été engagées dans le cadre du programme GeoCompostelle qui a débuté fin 2018 et qui se termine fin 2021. Ce programme mené au LISST (UMR-CNRS 5193) est co-financé par l'Europe et la région Occitanie dans le cadre de ses projets Readynov (innovation). Un premier volet autour de la recherche action vise à construire une plateforme collaborative pour la mise en réseau des propriétaires et gestionnaires des 78 composantes du bien en série «Chemins de Saint-Jacques-de-Compostelle en France » inscrit depuis 1998 sur la Liste du patrimoine mondial. Un deuxième volet autour de la recherche académique vise à améliorer les connaissances sur les mobilités vers Compostelle et l'impact de ces circulations sur les territoires traversés.

2. Nous considérons comme « image géographique » tout objet visuel permettant d'améliorer le degré de connaissance géographique. En ce sens, les cartes, les photos, les croquis, les schémas, les documents vidéo, entre autres, constituent des supports qui, en venant appuyer l'analyse classique sur la base de texte et de discours, peuvent contribuer à une meilleure compréhension de l'espace géographique.

3. Plus qu'un itinéraire visible sur l'espace traversé, matérialisé par un balisage spécifique et une signalétique directionnelle voire informationnelle, le chemin marque le territoire. Son empreinte va bien au-delà des circulations observables et des traces laissées par les pèlerins-cheminants. Il façonne le territoire en revitalisant l'offre de commerces et de services, en stimulant les aménagements ou encore les projets de développement.

4. Entretiens réalisés de novembre 2018 à septembre 2019.

5. En France, cinq grandes voies structurent l'itinérance vers Compostelle : la voie du Puy-enVelay, la voie d'Arles qui se divise en la voie du Piémont Pyrénéen, la voie de Vézelay et la voie de Tours. Aux côtés de ces principaux axes de circulation qui convergent vers l'Espagne, on assiste à 
un développement important du nombre de variantes et d'itinéraires de liaison, qui permettent de connecter ces différentes voies.

6. Quelques repères sur la commune : 1349 habitants (INSEE 2017), valeur stable ; 45 hbts $/ \mathrm{km}^{2}$; plus de 20000 marcheurs par an.

\section{RÉSUMÉS}

Cet article vise à questionner le processus de construction d'une exposition sur les chemins de Compostelle basée sur la mobilisation et la production d'images géographiques associées aux discours. À partir des discours collectés auprès des marcheurs et des acteurs du développement qui facilitent leurs mobilités, comment les images - ici géographiques - sont-elles élaborées et comment favorisent-elles la production d'un savoir scientifique et sa vulgarisation?

This article aims to question the construction process of an exhibition dedicated to the analyses of pilgrims' mobilities on the Way of Saint James in France, as well as their impact on the territories crossed by this pathways. Based on narratives collected from walkers and development actors who facilitate their mobility, this paper intends to answer the following questions: (i) how are geographical images such as maps, pictures and sketches are elaborated; (ii) how do they favour the production of scientific knowledge and, (iii) more broadly, the popularisation of scientific knowledge?

\section{INDEX}

Mots-clés : image géographique, discours, itinéraire culturel, circulation, développement rural Thèmes : Sur l'Image

Keywords : geographical image, narrative, cultural itinerary, circulation, rural development

\section{AUTEURS}

\section{DAVID LAGARDE}

David Lagarde, david.lagarde@univ-tlse2.fr, est chercheur postdoctoral au LISST (UMR-CNRS 5193), Université Toulouse - Jean Jaurès. Il a récemment publié :

- Lagarde D., Rayssac S., 2021. « Plus on y va, plus on a envie d'y aller ». Questionner l'envie de revenir sur les Chemins de Saint-Jacques de Compostelle », Belgeo [En ligne], n². URL: http:// journals.openedition.org/belgeo/49228 - DOI : https://doi.org/10.4000/belgeo.49228 - Lagarde D., 2019. Du refuge moyen-oriental à la dispersion mondiale. Structuration de l'exode syrien entre 2011 et 2016. Revue européenne des migrations internationales [En ligne], vol. 35, n 3-4. URL: http://journals.openedition.org/remi/13962 - DOI: https://doi.org/10.4000/remi.13962 - Lagarde D., 2019. Des colporteurs syriens dans la mondialisation. Mappemonde [En ligne], $\mathrm{n}^{\circ} 126$. URL: http://journals.openedition.org/mappemonde/1363 - DOI: https://doi.org/10.4000/ mappemonde. 1363 


\section{PATRICIA PANEGOS}

Patricia Panegos, patricia.panegos@univ-tlse2.fr, est Ingénieure enquêtes SHS, CNRS au LISST (UMR-CNRS 5193), Université Toulouse - Jean Jaurès. Elle a récemment publié :

- Duvernoy I., Gambino M., Bories O. Panegos P., 2018. L'expérience de la nature entre espaces urbains et non-urbains : résidence, pluri-résidence et mobilités dans les pratiques contemporaines de nature dans le sud-ouest de la France. 26e Colloquium of the Commission on the Sustainability of Rural Systems of the International Geographical Union, International Geographical Union (IGU). Santiago de Compostela, Espagne. 570 p.

\section{SÉBASTIEN RAYSSAC}

Sébastien Rayssac, sebastien.rayssac@univ-tlse2.fr, est maître de conférences au LISST-

Dynamiques Rurales (UMR-CNRS 5193), Université Toulouse - Jean Jaurès. Il a récemment publié : - Lagarde D., Rayssac S., 2021. « Plus on y va, plus on a envie d'y aller ». Questionner l'envie de revenir sur les Chemins de Saint-Jacques de Compostelle », Belgeo [En ligne], $\mathrm{n}^{\circ} 2$. URL: http:// journals.openedition.org/belgeo/49228 - DOI : https://doi.org/10.4000/belgeo.49228 - Barna R., Rayssac S., 2019. Adaptation de la haute montagne pour les randonneurs itinérants. L'accessibilité en questions. In Cholat F. (dir.), Tourisme(s) et adaptation(s) = Tourism and adaptation. Grenoble, Elya éditions, p. 235-245.

- Rayssac S. (dir.), 2017. Dossier : Cheminements, tourisme et gouvernance territoriale. Sud-Ouest Européen [En ligne], n 43, 124 p. URL: https://journals.openedition.org/soe/2494 - DOI: https:// doi.org/10.4000/soe. 2494 